\title{
THREE APPROACHES TO DESIGN ENGINEERING IN THE HEALTH DOMAIN: A SYSTEMIC PERSPECTIVE
}

\author{
Pannunzio, Valeria; Kleinsmann, Maaike; Snelders, Dirk \\ Delft University of Technology
}

\begin{abstract}
This paper proposes a categorization of existing approaches to healthcare-related engineering design based on systems thinking principles. Three existing approaches to healthcare-related engineering design are isolated which contribute differently to health systems fundamental purposes and interconnections. The three approaches are labeled as 'silent', 'overt', and 'convergent' health design. Each approach is defined and illustrated through an example. Following, practical advantages and disadvantages of each approach are discussed. A reflection is offered on the expected relevance of the convergent health design approach for present and future societal challenges in the health domain, and specifically on the recently growing field of e-health. Finally, open methodological challenges related to convergent health design are outlined and characterized as opportunities for future research.
\end{abstract}

Keywords: E-Health, Systems thinking, Systems Engineering (SE), Participatory design, Biomedical design

\section{Contact:}

Pannunzio, Valeria

Delft University of Technology

Product Innovation Management

The Netherlands

v.pannunzio@tudelft.nl

Cite this article: Pannunzio, V., Kleinsmann, M., Snelders, D. (2019) 'Three Approaches to Design Engineering in the Health Domain: A Systemic Perspective', in Proceedings of the 22nd International Conference on Engineering Design (ICED19), Delft, The Netherlands, 5-8 August 2019. DOI:10.1017/dsi.2019.106 


\section{INTRODUCTION}

On several occasions, engineering design disciplines have been indicated as a field of study that is relevant for the support of health systems at large (Reid et al., 2005; Ross, 2009). In particular, branches such as industrial and systems engineering (Valdez et al., 2010), information technologies (Payton, 2011), and human factors and ergonomics (Gurses, 2012) have been explicitly advocated as desirable allies of large scale health-related efforts. To maximize the effectiveness of the alliance between engineering design and healthcare, some authors have pointed out the need for design engineers to 'better understand the healthcare systems, including the users of that system, as the context into which specific design solutions must be delivered' (Clarkson et al., 2004). Ever since, new health-specific engineering design approaches, such as the Biodesign process (Yock et al., 2015) were formalized that include an element of 'direct immersion' in clinical contexts to identify unmet needs. These approaches, however, are more meant to support practitioners in developing propositions that 'fit' in existing health systems, than to obtain systemic impact through specific engineering design methods. Still recently, the limitations of current engineering design practices in generating large-scale health value were acknowledged, and new theoretical and empirical research initiatives were demanded taking a systems perspective on healthcare product and service design (Patou and Maier, 2017). The present contribution is to be regarded as a response to the theoretical side of this demand. Specifically, the paper introduces a categorization of existing approaches to health-related engineering design grounded on systems thinking principles as defined in Arnold and Wade (2015). The overall research question is formulated as: What are the differences in the way distinct design engineering approaches result in systemic impact in the health domain?

The paper is built as following. It starts with a description of the process followed to distinguish existing engineering design approaches and their differences in affecting health systems. Then, each of the approaches (silent health design, overt health design and convergent health design) is presented and demonstrated through an example. Finally, the approaches are compared and the relevance of convergent health design for upcoming societal challenges in the health domain is discussed.

\section{THE IMPACT OF ENGINEERING DESIGN ON HEALTH SYSTEMS}

To answer the research question, we follow a three-step process. First, health systems are characterised in their essential systemic outlook through a 'system test' (ibid.) performed on traditional definitions. Secondly, the possible roles of engineering design processes in this systemic outlook are inferred. Finally, existing approaches to engineering design are differentiated by characteristics that are expected, in principle, to determine the fulfilment of such roles.

\subsection{Health systems' systemic outlook: a two-way road}

In traditional systems thinking literature, systems are described to consists of elements, interconnections, and a function or purpose (Meadows, 2008). Following, we provide an essential structure for health systems in general, derived from traditional definitions, which can then be detailed and completed to describe specific health systems with their distinctive characteristics.

Health systems are traditionally defined as 'the combination of resources, organization, financing, and management that culminate in the delivery of health services to the population' (Roemer, 1991). As such, health systems inherently appear to include at least two elements, being (1) the health providers and (2) the health recipients - the latter being interchangeably identifiable as 'population', 'patients', 'customers', or 'citizens' depending on the framework of reference (van Olmen et al., 2010); and at least a purpose, being the delivery of health services to the population. Also, health systems include at least one interconnection between the two main elements, which are linked by mutual dependencies. Some frameworks, for example, identify health systems addressees as the 'demand-side', distinguishing them from the apparatuses producing and disseminating healthcare which are identified as the 'supply-side' (Cassels, 1995). In this sense, health providers and health recipients can be seen as being linked both by 'push' and 'pull' connections. The two-way nature of the interconnection is, also, reflected in the information flows travelling across the system, which move both from health providers towards health recipients (e.g. in the case of doctors informing patients about diseases) and from health recipients towards health providers (e.g.in the case of patients describing symptoms to their doctor).

Both of these kinds of information exchanges can be either direct (e.g. as in-person interactions) or derived (e.g. through the use of e-health solutions). Altogether, the inputs departing from the health 
recipients towards health systems can be seen as inherently afferent to the domain of health research, defined as 'the people, institutions, and activities whose primary purpose is to generate high-quality knowledge that can be used to promote, restore, and or maintain the health status of populations.' (WHO, 2018). Conversely, the inputs departing from health providers to health recipients can be seen as afferent to the domain of health practice or health services, defined as 'all services that have as primary purpose the improvement of health' (van Olmen et al., 2010). The relation between health research and practice has been at the centre of long lasting academic debate (see e.g., Cabana et al., 1999; Grol and Grimshaw 2003; Green et al., 2009). Specifically, systemic dysfunctions in the interconnections between the two domains have been problematized as the 'knowing-doing gap' (figure 1), defined in clinical literature as 'the gap that exists between what we know works based on the best available evidence and what we clinically practice' (Umscheid and Brennan, 2015). The knowing-doing gap constitutes a large-scale issue in modern medicine, as it undermines the benefits that can be achieved through advances in medical science (Cochrane et al., 2007).

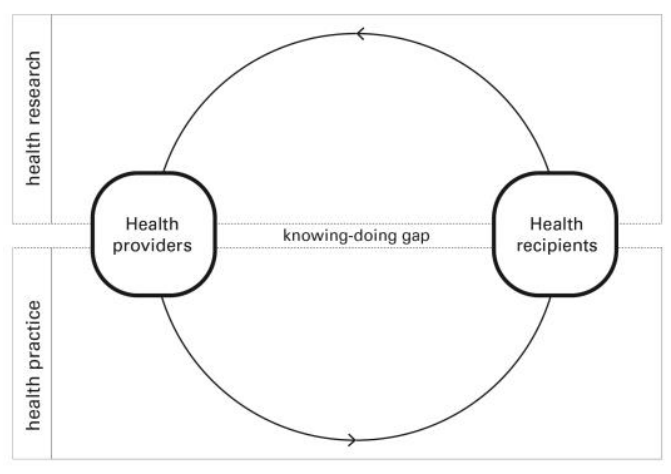

Figure 1. Visualization of health systems as a process flow

\subsection{Engineering design roles in health systems' systemic outlook: knowledge and practice}

In the second step of our process, the possible roles of engineering design processes in the previously defined systemic outlook are inferred. The most obvious role of engineering design processes within health systems is connected to the development of new health artefacts, here defined as any product, system, policy, protocol, or service aimed at the pursuit of health and wellbeing. Specifically, through the development of health artefacts, engineering design disciplines play a systemic role in the way health practice is performed, in the measure in which said health artefacts enable or mediate aspects of health practice. A possibly less obvious systemic role of engineering design within health systems is connected to the knowledge generated through engineering design processes. This knowledge can be divided at least in two categories; 1) knowledge obtained through health research methods, which adds to the disciplinary body of knowledge of the different branches of health research (e.g.; when clinical trials are performed as part of the development process of a new health artefact), and 2) knowledge obtained through design research methods, which adds to the disciplinary body of knowledge of engineering design (e.g.; when usability tests are performed as part of the development process of a new health artefact). The generation of both kinds of knowledge plays a systemic role in the functioning of health systems, since the former directly contributes to health research processes and the latter indirectly contributes to health practice processes. To sum up, thus, engineering design processes are inferred to play three possible systemic roles; 1) generating new health artefacts 2 ) generating new health knowledge through clinical research conducted as part of an engineering design process, and 3) generating new design knowledge through design research conducted as part of an engineering design process.

\subsection{Differentiation of engineering design approaches: three types of systemic impact}

Notably, not all kinds of design engineering processes play all of the previously defined systemic roles.

Specifically, engineering design approaches which do not result in the generation of one or more new health artefacts should not be considered to fulfil the first systemic role, while engineering design 
approaches which do not include health research as part of the new health artefact development process should not be considered to fulfil the second systemic role, and engineering design approaches which do not include design research as part of the new health artefact development process should not be considered to fulfil the third systemic role. Therefore, in principle, the three characteristics 1) generation of one or more new health artefacts; 2) presence of a design research processes; and 3) presence of a health research processes can be employed to surface differences in the way existing design engineering approaches impact on health systems.

For practical reasons, the case of engineering design processes which do not result in the generation of one or more new health artefacts will not be confronted in this paper; while constituting a conceptual and pragmatic possibility, we believe these cases are not only relatively rare, but also uninformative for the purpose of understanding systemic impact. As such, only approaches resulting in the generation of one or more new health artefacts will be examined, and each type of health design approach will be defined and compared in terms of 1) presence of a design research processes and 2) presence of a health research processes. These two characteristics can interplay with each other to generate four possible scenarios, listed in Table 1.

Table 1. Approaches to health-related design engineering

\begin{tabular}{|l|c|c|c|c|}
\hline & Silent health design & Overt health design & Convergent health design & / \\
\hline $\begin{array}{l}\text { Presence of a } \\
\text { design research } \\
\text { processes }\end{array}$ & No & Yes & Yes \\
\hline $\begin{array}{l}\text { Presence of a } \\
\text { health research } \\
\text { processes }\end{array}$ & No & No & Yes & Yes \\
\hline
\end{tabular}

The case of engineering design approaches that include health research and do not include design research will not be confronted in this paper, as such cases would essentially consist of clinical research initiatives, whose relevance for the design engineering community is expectedly null. Conversely, the remaining three approaches will be described and compared in detail. They will be referred to as 'silent', 'overt', and 'convergent' health design. We will illustrate each approach through an example, obtained by 1) using several combinations of keywords (such as 'design engineering healthcare') to search, in Google Scholar, for literature describing design engineering processes resulting in the development of new health artefacts; 2) selecting the examples that were more extensively documented from the point of view of the two characteristics of interest for the present research (presence of a design research processes and of a health research process).

\section{SILENT HEALTH DESIGN}

\subsection{Definition: health design that does not include any kind of research}

'Silent design' is a term introduced by Gorb and Dumas (1987) to denominate design activities that are not called design and are carried out by individuals who are not called designers. In the health domain, this definition applies to a whole era of health artefacts development. Silent health design has been, for instance, employed by a number of surgeons 'building their own wheel' and crafting new instrumentation inspired by everyday experience (Ailawadi et al., 2010), or by ambulance staff serendipitously creating new ways of fixing rescue information 'on-the-go' by writing on their own gloves (Pannunzio, 2016).

While silent health design process can be driven by in-depth clinical and contextual knowledge gained through practical experience, these are conducted by profiles who lack formal design education, and tend therefore not to follow a formal design research process. As such, the systemic impact of silent health design processes is limited to a one-directional flux of influence from health providers to health recipients (Figure 2).

Finally, silent health design may be conducted by one or more individuals afferent to a same discipline, and does not inherently require a multidisciplinary team composition, given the onedirectional, straightforward nature of the health artefact development process. 


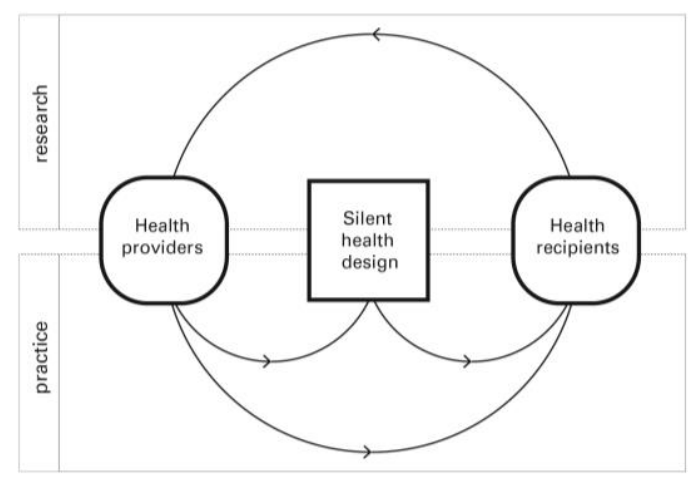

Figure 2. Visualization of a silent health design process, including input from health providers (in the form of clinical knowledge) and output towards health recipients (in the form of the designed health artefact)

\subsection{Example: the Metzenbaum scissors}

Myron Firth Metzenbaum (1876-1944), otolaryngologist, is the inventor of several health artefacts, including the "drop-ether anaesthesia" method and the bifocal operating spectacles. Yet, his fame is mainly due to the Metzenbaum scissors, one of the most ubiquitous tools in modern organ operations. This health artefact has been described to have arisen out of sheer practical necessity; according to Ailawadi et al. (2010), Metzenbaum himself had rather small hands, leading to difficulties in executing tonsillectomies. He thus had the idea of crafting scissors with an increased shank-to-blade ratio, allowing for more precise manoeuvring. According to Metson (1994), Metzenbaum explicitly avoided patenting his inventions, and surgical instrument companies of his time spontaneously started including the new scissors in their catalogues, resulting in widespread clinical adoption. The invention of the Metzenbaum scissors satisfies all of the conditions for silent health design as defined in Table 1; Metzenbaum did not follow any design research or clinical research process, and all that was necessary for the health artefact development was its conception and production.

\section{OVERT HEALTH DESIGN}

\subsection{Definition: health design that includes design research}

For Gorb and Dumas (1987), 'overt' design differs from silent design in that it is explicitly recognized as design and it is carried out by individuals who call themselves designers. In the health domain, cases of overt design abound and are increasingly reported in specialized literature. Bruce Archer's project in the early 1960s leading to the development of the 'King's Fund Bed', a predecessor of modern hospital beds, is considered as one of the first examples of design in the health domain (Park, 2015). Being conducted by professionals who hold formal design education, overt health design projects typically include a design research process. As such, overt health design processes include at least two kinds of specialized knowledge; 1) knowledge that is 'imported' from clinical disciplines; and 2) design knowledge that is generated during the internally conducted design research process (Figure 3).

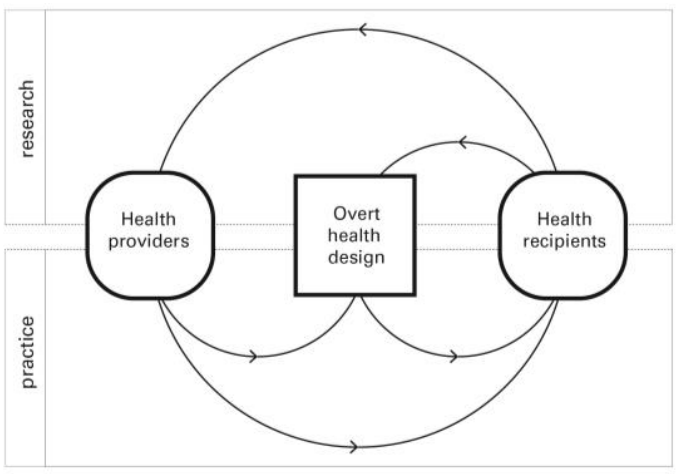

Figure 3. Visualization of an overt health design process, including input from health providers (in the form of existing clinical knowledge), input from health recipients (in the form of design-generated knowledge), and output towards health recipients (in the form of the designed health artefact) 


\subsection{Example: rehabilitation device for cerebral palsy patients}

Weightman et al. (2009) extensively describe the engineering design process leading to the development of two rehabilitation devices, including a computer game, for children with cerebral palsy. Target users, including children, parents and caregivers, and clinical experts such as medical doctors and paediatric physiotherapists were involved in the design process. As such, the process included both specialized clinical knowledge (such as notions on the therapeutic benefits of exercise for children with cerebral palsy) and design-generated knowledge on the users and their context (such as the notion that the favoured TV shows among target group children were Scooby Doo and The Simpsons). The rehabilitation devices were evaluated and iterated upon through qualitative and quantitative evaluation of aspects such as comfort, enjoyment and aesthetics.

\section{CONVERGENT HEALTH DESIGN}

\subsection{Definition: health design that includes both health and design research}

'Convergent' health design is described as a third approach to the development of evidence-based health artefacts, distinguished both from silent and overt health design. As in overt health design, the process is called design and is conducted by formally trained designers. Also, this approach, too, integrates both 'imported' clinical knowledge and 'self-generated' design knowledge as input for the design process. This approach, however, includes structured clinical research, resulting in the creation of new health knowledge that can then be 'fed back' to health providers (Figure 4). Because both a design and a research process are followed at the same time, convergent health design can be considered as a particular form of Research Through Design (Zimmerman et al., 2007), and perhaps more specifically, as a form of Design-Inclusive Research as described in Horváth (2007).

A feature of particular interest in the convergent health design approach is the collection and analysis of clinically relevant data as part of the health artefact evaluation. The presence of this clinical, datadriven component facilitates the distinction between convergent and overt health design as previously defined; in the described overt health design example, for instance, no clinical study was executed to evaluate the rehabilitation devices, which were tested through 'non-clinical' parameters alone.

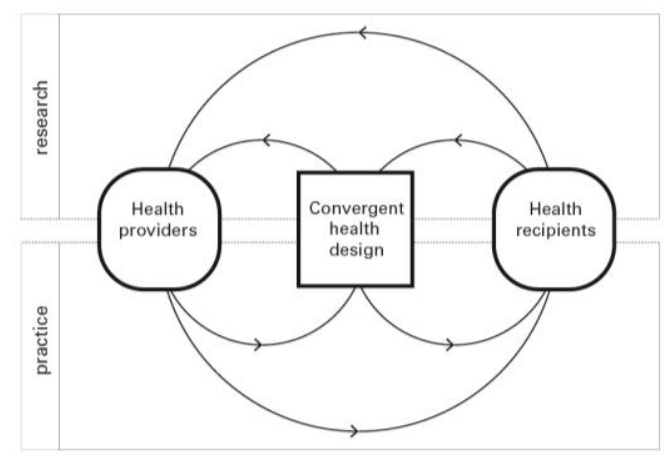

Figure 4. Visualization of a convergent health design process, including input from health providers (in the form of specialized knowledge), input from health recipients (in the form of design-generated knowledge), output towards health recipients (in the form of the designed artefact) and output towards health providers (in the form of new health knowledge generated through clinical research)

\subsection{Example: Statin Choice, a decisional aid for statin treatment}

A research group at the Mayo Foundation developed a decision aid, named Statin Choice, for patients with diabetes who were considering using statins to reduce their cardiovascular risk. The project, described extensively in Montori et al. (2007) and Breslin et al. (2008), was led by a multidisciplinary team including formally trained designers and clinical researchers. A structured design process was followed that included both 'imported' clinical knowledge (such as notions on the effectiveness of statins treatment in reducing cardiovascular risk in diabetic patients) and 'self-generated' design knowledge (such as notions derived through observations of patients-clinician interactions while deciding about initiating the treatment). The decisional aid was evaluated in a randomized trial against a standard educational pamphlet, which concluded that the use of Statin Choice as a decisional aid was associated with 'apparently greater' statin adherence at three months (Weymiller et al., 2007). 


\section{DISCUSSION}

\subsection{Approaches comparison}

The present research includes several limitations. Firstly, the process resulting in the identification of the three approaches is purely conceptual, and empirical research methods might surface entirely different types of approaches. Secondly, the identification and examination of the approaches concerns past projects, and does not necessarily provide insights on how to organize future enterprises so to obtain the desired kind of systemic impact. To make any considerations of this nature, further research would be required including careful examination of a larger volume of health design projects of each kind. However, a few preliminary considerations can be made on the practical advantages and disadvantages we can expect to be associated with each approach regarding process complexity and outcomes characteristics.

A practical advantage of silent health design when compared to the other two approaches is its relative simplicity and straightforwardness, resulting in faster and cheaper health artefact development process. However, the approach presents the disadvantage of relying entirely on the creativity and initiative of someone holding first-hand experience in the clinical field. While such occurrences should certainly be welcomed, they should not be considered as a formal responsibility of health providers who are normally trained to follow protocols, rather than to be innovative. Limiting health products and services development to such serendipitous events would result in missing on fruitful opportunities for innovation that can be obtained by having professionals who are 'trained' in product or service development to employ their skills in the clinical domain.

A practical advantage of overt health design when compared to the other two approaches is its explicit inclusion of an understanding on users and their context in the design process, while still affording a relative process agility compared to convergent health design. However, expected disadvantages of this approach include the lack of a strong clinical evidence base, possibly affecting outcomes quality in terms of; 1) clinical performance, and 2) eagerness of health providers at large to adopt the developed health artefact in clinical practice.

Finally, a practical advantage of convergent health design when compared to the other two approaches is its characteristic of providing a 'closed loop' of knowledge transfer between health providers and recipients (Figure 3), allowing for fruitful influences in both directions. However, expected disadvantages of this approach include a uniquely high degree of complexity, due to; 1) the need for close multidisciplinary alignment between design engineers, health researchers, health recipients and possibly other stakeholders, and 2) the need for long and costly clinical research procedures. In short, while none of the described approaches should be considered to be preferable per se, it is defended that each should be considered to affect health systems differently. Depending on the challenge and on the specific characteristics of the targeted health system at a certain moment in time, different engineering design approaches are expected to prove more beneficial than others. As such, the distinction between the three approaches constitutes a step forward in the theoretical understanding of the contribution of design engineering to the health domain from a systemic perspective. It is, finally, important to observe that the three approaches do not necessarily exclude each other, but can peacefully coexist, even within a same organization or research institution.

\subsection{Convergent health design and future societal challenges in the health domain}

As previously touched, a key advantage of the convergent health design approach is its enabling of a loop of knowledge circulation between health providers and health recipients. Through convergent health design, the knowledge on 'health users' and their context that is generated through engineering design research is not only embodied in the health artefact, but can be propagated and built upon by other health professionals and design engineers. Contextual knowledge preservation and transfer constitutes a desirable characteristic of all 'knowledge-intensive' design projects, in which, often, new specialized knowledge is generated yet remains undisclosed (Stappers, 2007). Conversely, feeding such knowledge back to the disciplinary sources determines, in the health domain, the potential for convergent health design to bridge the knowing-doing gap as characterized in section 2.1. Such a bridging can lead to concrete societal advantages, both in the present and future. In the present, a wider application of convergent health design approaches holds the promise to result in increased adoption of evidence-based interventions both by the health providers and health recipients. As noted by Damschroder et al. (2009) "many interventions found to be effective in health services research 
studies fail to translate into meaningful patient care outcomes across multiple contexts. In fact, some estimates indicate that two-thirds of organizations' efforts to implement change fail'. In the future, foreseeable societal and sociotechnical challenges appear to be particularly suitable ground for convergent health design. E-health and decentralized medicine, especially, constitute domains in which a balanced integration between user-centeredness, technical soundness and clinical rigour appears to be fundamental for ethical, safe and effective innovation. As noted by Jones (2013), 'the decentralized 'future of medicine' scenarios articulate radical changes in technology but fail to address changes in cultural meaning (...). If patients are forced by economic changes to trust a technology instead of a physician, the ethics of "brave new healthcare" scenario become socially problematic.

Considering the ongoing shift towards chronic, non-communicable disease as a global cause of morbidity and mortality (Murray and Lopez, 2013), and the consequent growing need for e-health based, decentralized solutions for delivering health to the population (Kruk et al., 2015), convergent health design becomes a strategic area for future-oriented research. Specifically, at the CardioLab, one of the Delft Design Labs at the Industrial Design Engineering faculty of the TU Delft, we intend to investigate the potential of convergent health design for advancing cardiovascular prevention research and practice, with a specific focus on e-health technologies. By developing integrated smart solutions for cardiovascular prevention through participatory design methods that involve both health providers and health recipients, we expect not only to generate new health artefacts, but also to generate both new design and health knowledge, which will complement and enhance knowledge gained through mono-disciplinary efforts. Such expectations are consistent with scholars such as Green (2008), who conceives 'a future in which the cumulative, building-block tradition of evidence-based medicine from highly controlled trials would be complemented by a parallel development and support of a tradition of participatory research and evaluation conducted in practice settings' and as Sharp et al. (2016), who elaborate on the importance of convergence (the 'integration of insights and approaches from historically distinct scientific and technological disciplines') for the future of health.

\subsection{The way forward for convergent health design}

Recently, first attempts at large-scale, strategic prioritizations of approaches afferent to convergent health design have been reported. The World Health Organization released a global strategy executive summary for the time window 2016-2026 revolving around the recognition that the 'benefits of a people-centred and integrated approach are well documented: increased delivery efficiency, decreased costs, improved equity in uptake of service, better health literacy and self-care, increased satisfaction with care, improved relationships between patients and their care providers, and an improved ability to respond to health-care crises.' (WHO, 2015).

Yet, the way ahead is long and uncertain; convergent health design being a relatively recent approach to health artefact development, further research is required to reach a thorough understanding of the approach own methodological challenges and ways to overcome them. The development of new, effective ways to enable mutual understanding between design engineers and health researchers, for example, or to orchestrate data collection infrastructures that can feed both clinical and design research, would constitute invaluable progress in building a solid and effective tradition of convergent health design. Regarding this latter challenge, we can already find inspiration in the field, for instance in studies such as 'Designing for co-responsibility to improve patient engagement, a data-enabled approach' (Formulier Voor Medisch-Ethische Beoordeling en Registratie, 2018) from Ineke Neutelings and Jos-marien Jansen, in which sensor data from bariatric patients was collected both to conduct design research and to investigate the clinical relevance of the data itself. Other stimulating examples in which data explorations led to convergent value-creation scenarios can be found in van Kollenburg et al. (2018), who explored the value of self-tracked data for end users, healthcare professionals and design researchers through innovative design research approaches. In this spirit, this paper constitutes an open call to all design engineers working in the health domain to recognize the extent of their own role within healthcare systems, identify common challenges, share insights and best practices, and foster mutually beneficial interdisciplinary dialogue.

\section{CONCLUSION}

This paper offered a description of three different engineering design approaches to the development of health artefacts. The approaches were characterized as 'silent', 'overt', and 'convergent' health 
design. Each approach was defined and illustrated through an example, and practical advantages and disadvantages of each approach were discussed. A reflection was offered on the relevance of convergent health design for upcoming societal challenges in the health domain. Finally, methodological challenges related to convergent health design were outlined and characterized as opportunities for future research.

\section{REFERENCES}

Ailawadi, G., Nagji, A.S. and Jones, D.R. (2010), “The legends behind cardiothoracic surgical instruments", The Annals of thoracic surgery, Vol. 89 No. 5, pp. 1693-1700. http://dx.doi.org/10.1016/j.athoracsur.2009.11.019.

Arnold, R.D. and Wade, J.P. (2015), “A definition of systems thinking: a systems approach”, Procedia Computer Science, Vol. 44, pp. 669-678. https://doi.org/10.1016/j.procs.2015.03.050

Breslin, M., Mullan, R.J. and Montori, V.M. (2008), "The design of a decision aid about diabetes medications for use during the consultation with patients with type 2 diabetes", Patient education and counseling, Vol. 73 No. 3, pp. 465-472. https://doi.org/10.1016/j.pec.2008.07.024

Cabana, M.D., Rand, C.S., Powe, N.R., Wu, A.W., Wilson, M.H., Abboud, P.-A.C. and Rubin, H.R. (1999), "Why don't physicians follow clinical practice guidelines?: A framework for improvement", Journal of the American Medical Association, Vol. 282 No. 15, pp. 1458-1465. https://doi.org/10.1001/jama.282.15.1458

Cassels, A. (1995), "Health Sector reform: Some key issues in less developed countries", Journal of International Development, Vol. 7 No. 3, pp. 329-348. https://doi.org/10.1002/jid.3380070303

Clarkson, P.J., Buckle, P., Coleman, R., Stubbs, D., Ward, J., Jarrett, J., Lane, R. and Bound, J. (2004), "Design for patient safety: a review of the effectiveness of design in the UK health service", Journal of Engineering Design, Vol. 15 No. 2, pp. 123-140. https://doi.org/10.1080/09544820310001617711

Cochrane, L.J., Olson, C.A., Murray, S., Dupuis, M., Tooman, T. and Hayes, S. (2007), "Gaps between knowing and doing: understanding and assessing the barriers to optimal health care", Journal of continuing education in the health professions, Vol. 27 No. 2, pp. 94-102. https://doi.org/10.1002/chp.106

Damschroder, L.J., Aron, D.C., Keith, R.E., Kirsh, S.R., Alexander, J.A. and Lowery, J.C. (2009), “Fostering implementation of health services research findings into practice: a consolidated framework for advancing implementation science", Implementation science, Vol. 4 No. 1, p. 50. https://doi.org/10.1186/1748-5908-4-50

Formulier Voor Medisch-Ethische Beoordeling en Registratie (2018), "Designing for co-responsibility to improve patient engagement, a data-enabled approach". [online] Centrale Commissie Mensgebonden Onderzoek. Available at:

(https://www.toetsingonline.nl/to/ccmo_search.nsf/fABRpop?readform\&unids=95392C4311CF1029C125 8339001A8D01) (18.03.2019).

Gorb, P. and Dumas, A. (1987), "Silent design”, Design studies, Vol. 8 No. 3, pp. 150-156. https://doi.org/10.1016/0142-694x(87)90037-8

Green, L.W. (2008), "Making research relevant: if it is an evidence-based practice, where's the practice-based evidence?", Family practice, Vol. 25, pp. i20-i24. https://doi.org/10.1093/fampra/cmn055

Green, L.W., Ottoson, J.M., Garcia, C. and Hiatt, R.A. (2009), "Diffusion theory and knowledge dissemination, utilization, and integration in public health", Annual review of public health, Vol. 30 No. 1, pp. 151-174. http://dx.doi.org/10.1146/annurev.publhealth.031308.100049.

Grol, R. and Grimshaw, J. (2003), "From best evidence to best practice: effective implementation of change in patients' care", The Lancet, Vol. 362 No. 9391, pp. 1225-1230. https://doi.org/10.1016/s01406736(03)14546-1

Gurses, A.P., Ozok, A.A. and Pronovost, P.J. (2012), "Time to accelerate integration of human factors and ergonomics in patient safety", BMJ Quality \& Safety, Vol. 21 No. 4, pp. 347-351. https://doi.org/10.1136/bmjqs-2011-000421

Horváth, I. (2007), "Comparison of Three Methodological Approaches of Design Research", Proceedings of ICED 2007, the 16th International Conference on Engineering Design, Paris, 28.-31.08.2007, The Design Society 2007, Glasgow, pp. 1-11.

Jones, P. (2013), Design for care: Innovating healthcare experience". Rosenfeld Media, Brooklyn, New York.

Kruk, M.E., Nigenda, G. and Knaul, F.M. (2015), "Redesigning primary care to tackle the global epidemic of noncommunicable disease", American journal of public health, Vol. 105 No. 3, pp. 431-437. https://doi.org/10.2105/ajph.2014.302392

Meadows, D.H. (2008), Thinking in systems: A primer, Earthscan, London.

Metson, R. (1994), "Myron F. Metzenbaum, MD: Innovative surgeon, caring physician", Otolaryngology - Head and Neck Surgery, Vol. 110 No. 6, pp. 477-481. https://doi.org/10.1177/019459989411000601

Montori, V.M., Breslin, M., Maleska, M. and Weymiller, A.J. (2007), "Creating a conversation: insights from the development of a decision aid", PLoS medicine, Vol. 4 No. 8, p. e233.

https://doi.org/10.1371/journal.pmed.0040233 
Murray, C.J.L. and Lopez, A.D. (2013), "Measuring the global burden of disease", New England Journal of Medicine, Vol. 369, pp. 448-457. https://doi.org/10.1056/NEJMra1201534

Pannunzio, V. (2016), "Effortless Interactions for Emergency Care”, Delft University of Technology. https://doi.org/10.31237/osf.io/62n85

Park, J.H. (2015), "Health care design: current and potential research and development", Design Issues, Vol. 31 No. 1, pp. 63-72. https://doi.org/10.1162/desi_a_00310

Patou, F. and Maier, A. (2017), "Engineering value-effective healthcare solutions: A systems design perspective". in DS 87-3 Proceedings of the 21st International Conference on Engineering Design (ICED 17) Vol 3: Product, Services and Systems Design, Vancouver, Canada, 21-25.08.2017. pp. 031-040.

Payton, F.C., Pare, G., LeRouge, C. and Reddy, M. (2011), "Health Care IT: Process, People, Patients and Interdisciplinary Considerations", Journal of the Association for Information Systems, Vol. 12 No. 2, p. I. https://doi.org/10.17705/1jais.00259

Reid, P.P., Compton, W.D., Grossman, J.H. and Fanjiang, G. (2005), Building a better delivery system: a new engineering/health care partnership, National Academies Press. Washington, DC. https://doi.org/10.17226/11378

Roemer, M.I. (1991), National health systems of the world, Oxford university press, New York.

Ross, D.A. (2009), "Process engineering: A necessary step to a better public health system", Information Knowledge Systems Management, Vol. 8 No. 1-4, pp. 299-309.

Sharp, P., Hockfield, S. and Jacks, T. (2016), Convergence: the future of health. The Convergence Revolution. Cambridge, MA.

Stappers, P.J. (2007), "Doing design as a part of doing research”, Design research now, pp. 81-91. https://doi.org/10.1007/978-3-7643-8472-2_6

Umscheid, C.A. and Brennan, P.J. (2015), "Incentivizing "structures" over "outcomes" to bridge the knowingdoing gap”. JAMA internal medicine, Vol. 175 No. 3, pp. 354-355. https://doi.org/10.1001/jamainternmed.2014.5293

Valdez, R.S., Ramly, E. and Brennan, P.F. (2010), Industrial and systems engineering and health care: critical areas of research, Agency for Healthcare Research and Quality, Rockville, MD.

van Olmen, J., Criel, B., Van Damme, W., Marchal, B., Van Belle, S., Van Dormael, M., Hoerée, T., Pirard, M. and Kegels, G. (2010), “Analysing health systems to make them stronger”, Studies in Health Services Organisation \& Policy, No. 27.

Van Kollenburg, J., Bogers, S., Rutjes, H., Deckers, E., Frens, J. and Hummels, C. (2018), "Exploring the Value of Parent Tracked Baby Data in Interactions with Healthcare Professionals: A Data-Enabled Design Exploration”, Proceedings of the 2018 CHI Conference on Human Factors in Computing Systems (p. 297). https://doi.org/10.1145/3173574.3173871

Weightman, A.P.H., Preston, N., Holt, R., Allsop, M., Levesley, M. and Bhakta, B. (2010), "Engaging children in healthcare technology design: developing rehabilitation technology for children with cerebral palsy", Journal of Engineering Design, Vol. 21 No. 5, pp. 579-600. https://doi.org/10.1080/09544820802441092

Weymiller, A.J., Montori, V.M., Jones, L.A., Gafni, A., Guyatt, G.H., Bryant, S.C., Christianson, T.J., Mullan, R.J. and Smith, S.A. (2007), "Helping patients with type 2 diabetes mellitus make treatment decisions: statin choice randomized trial", Archives of internal medicine, Vol. 167(10), pp. 1076-1082.

WHO (2018), Health Research Systems Analysis (HRSA) concepts and indicators [online] WHO. Available at: (http://www.who.int/rpc/health_research/concepts/en/) (9.11.2018).

WHO (2015), WHO global strategy on integrated people-centred health services 2016-2026 [online] WHO. Available at: (http://africahealthforum.afro.who.int/firstedition/IMG/pdf/the_global_strategy_for_integrated_people_centred_health_services.pdf) (2-.03.2019)

Yock, P.G., Zenios, S., Makower, J., Brinton, T.J., Kumar, U.N., Watkins, F.J., Denend, L., Krummel, T.M. and Kurihara, C.Q. (2015), Biodesign: the process of innovating medical technologies. Cambridge University Press. Cambridge, UK.

Zimmerman, J., Forlizzi, J. and Evenson, S. (2007), "Research through design as a method for interaction design research in HCI", Proceedings of the SIGCHI conference on Human factors in computing systems (pp. 493-502). https://doi.org/10.1145/1240624.1240704

\section{ACKNOWLEDGMENTS}

We gratefully acknowledge Ineke Neutelings, Jiwon Jung, Peter Lovei, Pascale Carayon and the anonymous peer reviewers for the inspiration, support and valuable feedback. 